hep-ph/9804313

NEIP-98-001

HD-THEP-98-14

DSF-1/98

MAP-221

\title{
Renormalization Group Naturalness of GUT Higgs Potentials
}

\author{
B. C. Allanach ${ }_{a}^{1}$, G. Amelino-Camelia 2, , O. Philipsen ${ }_{c}^{3}$, O. Pisanti ${ }_{d}^{4,5}$, and L. Rosa ${ }_{e}^{4}$ \\ 1. Department of Particle Physics, Rutherford Appleton Laboratory, \\ Chilton, Didcot, Oxon, OX11 OQX, UK \\ 2. Institut de Physique, Université de Neuchâtel, \\ rue Breguet 1, CH-2000 Neuchâtel, Switzerland \\ 3. Institut für Theoretische Physik, Universität Heidelberg, \\ Philosophenweg 16, 69120 Heidelberg, Germany \\ 4. Dipartimento di Scienze Fisiche, Università di Napoli, and INFN, Sez. di Napoli, \\ Mostra d'Oltremare, Pad.19, I-80125 Napoli, Italy \\ 5. Kellogg Radiation Laboratory, California Institute of Technology, \\ Pasadena, CA 91125, USA
}

\begin{abstract}
We analyze the symmetry-breaking patterns of grand unified theories from the point of view of a recently-proposed criterion of renormalization-group naturalness. We perform the analysis on simple non-SUSY SU(5) and SO(10) and SUSY SU(5) GUTs. We find that the naturalness criterion can favor spontaneous-symmetry-breaking in the direction of the smallest of the maximal little groups. Some differences between theories with and without supersymmetry are also emphasized.
\end{abstract}
a) bca@hep.phys.soton.ac.uk
b) giovanni.amelino-camelia@cern.ch
c) o.philipsen@thphys.uni-heidelberg.de
d) pisanti@na.infn.it
e) rosa@na.infn.it 


\section{Introduction}

A large number of grand unified theories (GUTs) has already been discussed in the literature, and, assuming that the GUT idea is successful, we are confronted with the fact that the constraints imposed by the low-energy data are not sufficient to select the correct GUT. Certain additional constraints on the structure of a GUT emerge if one requires that it be a plausible candidate as low-energy effective description of a more fundamental theory (for example including gravity). In particular, in Ref. [1] it was observed that one could study the RG equations describing the running of the parameters of the Higgs potential between $M^{*}$, denoting the scale (possibly given by the Planck scale $M_{P} \sim 10^{19} \mathrm{GeV}$ ) where the GUT emerges as low-energy effective theory, and the GUT scale $M_{X}$ (the scale where the low energy couplings unify). This could establish whether or not a given pattern of SSB (spontaneous symmetry breaking) can be naturally obtained. In this context, we define a symmetry breaking direction to be natural if it corresponds to a large volume of parameter space at $M^{*}$. For example, if a strongly attractive fixed point was found within a region of the space of parameters of the Higgs potential that corresponds to a certain SSB pattern, one would then expect that in running toward the infra-red direction, the Higgs parameters would approach their fixed point values (forcing the corresponding SSB pattern) quite independently of the input parameters at the scale $M^{*}$.

Importantly, this $R G$ naturalness criterion concerns one of the non-predictive aspects of GUTs, i.e. the SSB pattern. Even after selecting the matter (Higgs) content, most GUTs may break in several different ways depending on which vacuum expectation values are acquired by the Higgs fields. If, as ordinarily assumed, the direction of SSB is determined by the global minimum of the Higgs potential, the symmetry-breaking pattern of a GUT only depends upon the input parameters in the Higgs potential of the model. While we are discussing here the first step of GUT SSB, similar RG naturalness considerations could of course be applied to other steps of SSB.

In Ref. [2] the criterion of RG naturalness has already been applied to the study of the first step of SSB of a SUSY (supersymmetric) SU(5) GUT. Interestingly, an infra-red fixed point was identified analytically, and it was found to be located at the boundary between the region of Higgs parameter space corresponding to unbroken $\mathrm{SU}(5)$ and the region corresponding to the breaking of $\mathrm{SU}(5)$ to the Standard Model gauge group $G_{S M} \equiv S U(3) \otimes S U(2) \otimes U(1)$. Also motivated by the fact that the presence of a fixed point at the boundary between two SSB regions may not be typical, we intend to illustrate a wider range of possible scenarios for the outcome of a RG naturalness analysis. We do this by studying the first step of SSB of two additional examples, i.e. non-SUSY $S U(5)$ and $S O(10)$ GUTs. In order to discuss the role that SUSY might play in RG naturalness analyses, we also briefly review the results of Ref. [2].

In the remainder of this introduction we mention a few other RG approaches which have been used in the study of GUTs, and we comment on the relation between these approaches and the type of approach advocated here. Let us start by mentioning those studies (see, e.g., Ref. [3] and references therein) that have established how predictions for the low-energy values of certain quantities can be obtained from the infra-red structure of the relevant RG equations. While these studies do not always assume grand unification, the fact that the values of (low-energy) parameters can be derived from the structure of the RG equations is encouraging for our attempt to derive the SSB pattern in a similar way.

RG techniques have also already been applied to the study of GUT-scale physics; notably in the context of certain analyses of stability [4, 5]. While the actual formulae one ends up studying are in some cases the same, the emphasis in stability analyses is quite different from the one of the present article, especially since the stability characterizes a theory at the GUT scale, whereas here one is interested in the physics between the scale $M^{*}$ and the 
GUT scale.

Another example of RG ideas applied to GUT-scale physics is given by the studies of finite GUTs [6]. Some of the finite GUTs that correspond to an IR fixed point of the RG equations for the Higgs parameters might provide a good starting point for the construction of an RG natural GUT; however, dedicated analyses are necessary since the literature on finite GUTs has often not considered SSB and the parameters of the Higgs potential in detail.

Finally, we should mention the idea of radiative breaking [7], in which the symmetrybreaking term is induced directly by the RG running, rather than being included by hand in the Higgs potential at a given scale. This idea is based on an intuition that is very close to the one behind the criterion of RG naturalness. The two proposals primarily differ in that the former restricts the analysis to models in which the symmetry-breaking term is directly induced by the RG running, whereas the latter includes models in which a non-vanishing symmetry-breaking term is already introduced at the scale $M^{*}$ as long as SSB occurs in the direction favored by the RG running.

We now turn to explicit examples of GUT Higgs potentials. The analysis reported in the following sections should also illustrate the similarities and the differences between RG naturalness analyses and the above approaches concerning the use of $\mathrm{RG}$ equations in the study of grand unification.

\section{A SUSY SU(5) model}

Let us start, as anticipated, with a brief review of the results obtained in Ref. [2], where the criterion of RG naturalness was applied to the study of the first step of SSB of the minimal SUSY SU(5) GUT. This model involves the Higgs fields of the 24-dimensional irreducible representation (the adjoint), which are responsible for the first step of SSB, and it also includes $\underline{5}+\underline{\overline{5}}$ Higgs, which are used in the second SSB step. However, for simplicity in our analysis of the first SSB step we neglect the effects of the $\underline{5}+\underline{\overline{5}}$ Higgs. We therefore limit our analysis to the potentials involving the 24 Higgs. The superpotential is taken to be [8]

$$
W=\lambda_{1} \operatorname{Tr}\left(\Sigma^{3}\right)+\mu \operatorname{Tr}\left(\Sigma^{2}\right),
$$

where $\Sigma$ denotes the 24-dimensional superfield multiplet. We assume that SUSY breaking is explicit, via the "soft" SUSY-breaking terms in the potential

$$
V_{\text {soft }}=\left[\frac{m_{3}}{6} \operatorname{Tr}\left(\sigma^{3}\right)+m_{2}^{2} \operatorname{Tr}\left(\sigma^{2}\right)+\frac{M}{2} \lambda \lambda+\text { h.c. }\right]+m_{3 / 2}^{2} \operatorname{Tr}\left(\sigma^{\dagger} \sigma\right),
$$

where $\sigma$ represents the scalar component of $\Sigma$ and $\lambda$ denotes the $\mathrm{SU}(5)$ gaugino. The full Higgs potential relevant for the first step of SSB can be written as

$$
V=\left|\frac{\partial W}{\partial \Sigma_{i}}\right|^{2}+V_{\text {soft }}+\text { D-terms . }
$$

Based on hierarchy arguments [9] we expect $m_{3}, m_{3 / 2}, M \sim 1 \mathrm{TeV}$ and $m_{2} \sim 10^{11} \mathrm{GeV}$, while $\mu$ is a GUT scale parameterf expected to be of order $10^{16} \mathrm{GeV}$.

\footnotetext{
${ }^{1}$ In GUT-scale radiative-breaking scenarios one considers the possibility $\mu=0$, which is stable under the one-loop RG equations. In the present work we shall ignore this possibility. Its analysis would require a generalization of our study of the Higgs potential, not relying on the simplifications we achieve by assuming $\left|m_{i} / \mu\right| \ll 1$.
} 
It is convenient to consider the three independent combinations of parameters $\delta_{2} \equiv m_{2}^{2} / \mu^{2}$, $\delta_{3} \equiv m_{3} / \mu$ and $\delta_{3 / 2} \equiv m_{3 / 2} / \mu$, which allow to rewrite the soft-breaking potential as [2]

$$
V_{\text {soft }}=\frac{8 \mu^{4}}{27 \lambda_{1}^{2}} b F,
$$

where $b=0$ in the minimum preserving the full $S U(5)$ invariance, $b=30$ for the $G_{S M}$-invariant minimum, $b=20 / 9$ for the $S U(4) \otimes U(1)$-invariant minimum and

$$
F \equiv 3 \delta_{2}-\frac{1}{3 \lambda_{1}} \delta_{3}+\frac{3}{2} \delta_{3 / 2}^{2}
$$

Hence, the value of $F$ at the GUT scale $M_{X}$ determines the type of residual symmetry below $M_{X}$. If $F<0$ the $G_{S M}$-invariant minimum is the lowest one, while $S U(5)$ remains unbroken if $F>0$.

The one-loop RG equations may be easily derived [2] following the general prescriptions of Martin and Vaughn [10],

$$
\begin{aligned}
16 \pi^{2} \frac{d \lambda_{1}}{d t} & =3 \lambda_{1}\left(\frac{189}{40} \lambda_{1}^{2}-10 g^{2}\right) \\
16 \pi^{2} \frac{d \mu}{d t} & =2 \mu\left(\frac{189}{40} \lambda_{1}^{2}-10 g^{2}\right) \\
16 \pi^{2} \frac{d m_{3}}{d t} & =3\left[m_{3}\left(\frac{189}{40} \lambda_{1}^{2}-10 g^{2}\right)+\frac{189}{20} \lambda_{1}^{2} m_{3}+120 M \lambda_{1} g^{2}\right] \\
16 \pi^{2} \frac{d m_{2}^{2}}{d t} & =2\left[m_{2}^{2}\left(\frac{189}{40} \lambda_{1}^{2}-10 g^{2}\right)+\frac{63}{40} \lambda_{1} \mu m_{3}+20 M \mu g^{2}\right] \\
16 \pi^{2} \frac{d m_{3 / 2}^{2}}{d t} & =\frac{567}{20} \lambda_{1}^{2} m_{3 / 2}^{2}+\frac{21}{80} m_{3}^{2}-40 M^{\dagger} M g^{2} \\
16 \pi^{2} \frac{d g^{2}}{d t} & =\beta g^{4} \\
16 \pi^{2} \frac{d M}{d t} & =\beta g^{2} M
\end{aligned}
$$

where $t=\ln \left(q^{2} / M_{X}^{2}\right), q$ is the $\overline{M S}$ renormalization scale, $g$ is the gauge coupling and the one loop beta function, $\beta=2(S(R)-15)$, is determined by the sum over all the Dynkin indices of the fields in the theory, $S(R) . \beta=-8$ for our SUSY SU(5) model, which hosts the above mentioned Higgs sector plus $3(\underline{10} \oplus \underline{\overline{5}})$ representations corresponding to 3 Standard Model fermionic families (and superpartners).

To render the fixed point structure explicit, from (6-12) we form the following RG equations for dimensionless ratios

$$
\begin{aligned}
16 \pi^{2} \frac{d}{d t}\left(\frac{\lambda_{1}^{2}}{g^{2}}\right) & =6 g^{2}\left(\frac{\lambda_{1}^{2}}{g^{2}}\right)\left[\frac{189}{40}\left(\frac{\lambda_{1}^{2}}{g^{2}}\right)-10-\frac{\beta}{6}\right] \\
16 \pi^{2} \frac{d}{d t}\left(\frac{m_{3}}{M \lambda_{1}}\right) & =9 g^{2}\left[\left(\frac{m_{3}}{M \lambda_{1}}\right)\left[\left(\frac{\lambda_{1}^{2}}{g^{2}}\right) \frac{63}{20}-\frac{\beta}{9}\right]+40\right] \\
16 \pi^{2} \frac{d}{d t}\left(\frac{m_{2}^{2}}{M \mu}\right) & =g^{2}\left[-\beta\left(\frac{m_{2}^{2}}{M \mu}\right)+\frac{63}{20}\left(\frac{m_{3}}{M \lambda_{1}}\right)\left(\frac{\lambda_{1}^{2}}{g^{2}}\right)+40\right] .
\end{aligned}
$$



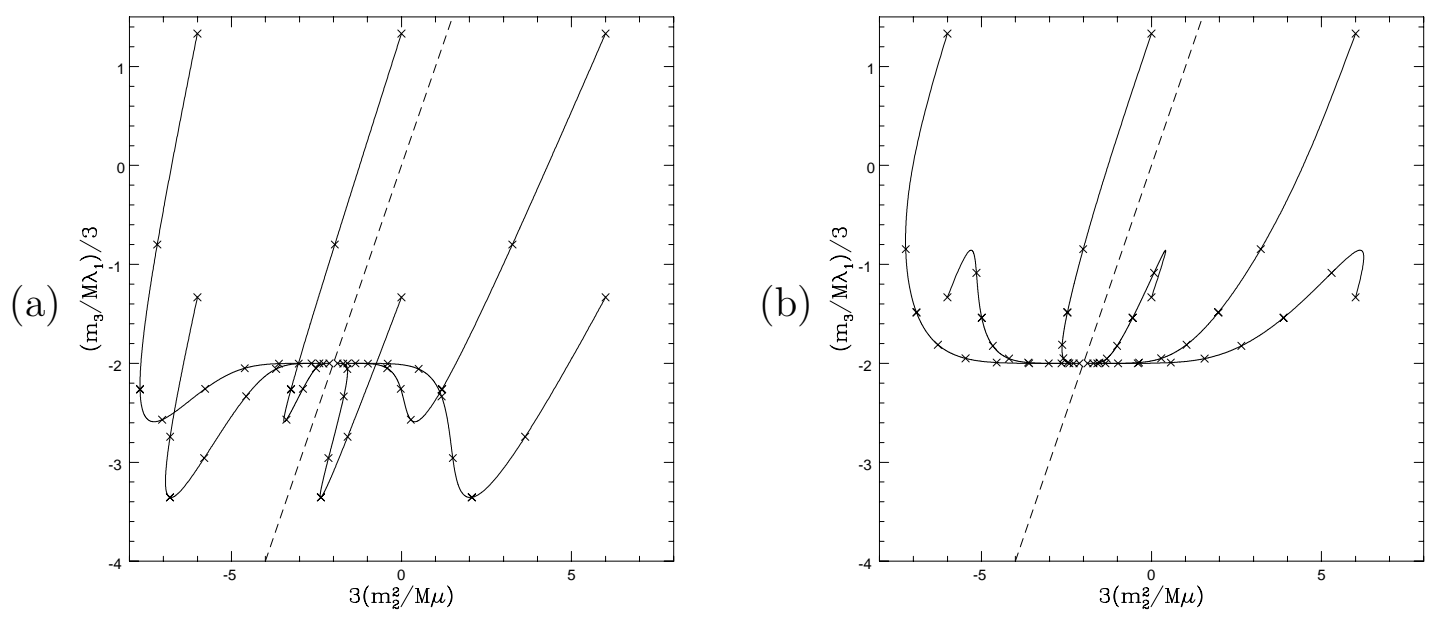

Figure 1: $R G$ flow between $M_{P}$ and $M_{X}$ of the soft SUSY-breaking parameters in SUSY $S U(5)$ with $\beta=-8$ for initial conditions with a) $\lambda_{1}\left(M^{*}\right)=0.3$ and $\left.b\right) \lambda_{1}\left(M^{*}\right)=2.0$. Every decrease of the scale by a factor $10^{3 / 2}$ is marked on the flow.

The right-hand side of this system of coupled equations vanishes for

$$
\left(\frac{\lambda_{1}^{2}}{g^{2}}\right)^{*}=\frac{40}{189}(10+\beta / 6), \quad\left(\frac{m_{3}}{M \lambda_{1}}\right)^{*}=-6, \quad\left(\frac{m_{2}^{2}}{M \mu}\right)^{*}=-\frac{2}{3}
$$

The fixed point described by Eq. (16) is a specific example of a more general class of fixed points identified in Ref. [11]. By linearizing (13-15) around the fixed point one easily finds that it is infra-red stable when $\beta<0$, as in the case of the SUSY SU(5) model considered here. For $\beta>0$, which can be achieved by adding more matter to the model, one would have a saddle point. Assuming $\delta_{3 / 2} \ll 1$, as implied by hierarchy arguments, we may neglect the second order contribution of order $\delta_{3 / 2}^{2}$, and $F$ is well approximated by

$$
F \approx 3 \delta_{2}-\frac{1}{3 \lambda_{1}} \delta_{3}=\frac{M}{\mu}\left[3 \frac{m_{2}^{2}}{M \mu}-\frac{1}{3} \frac{m_{3}}{M \lambda_{1}}\right],
$$

which is zero at the fixed point. Thus, starting at some scale $M^{*}$, e.g. the Planck scale, and running to the GUT scale, the Higgs parameters evolve towards values at the boundary $(F=0)$ between the region of parameter space corresponding to unbroken $\mathrm{SU}(5)$ and the region of parameter space corresponding to $\mathrm{SU}(5)$ breaking to $G_{S M}$.

We have also studied our RG equations numerically for the parameter values $M^{*}=M_{P}=$ $10^{19} \mathrm{GeV}, \mu\left(M^{*}\right)=10^{16} \mathrm{GeV}, M\left(M^{*}\right)=m_{3 / 2}\left(M^{*}\right)=10^{3} \mathrm{GeV}$. The gauge coupling is fixed by $g^{2}\left(M_{X}\right)=8 \pi / 5$ to ensure consistency of SUSY SU(5) unification of the Standard Model couplings with the low-energy experimental data. The running parameters $M$ and $\mu$ evolve slowly (they decrease by a factor of about $1 / 2$ between the Planck and the GUT scale); consequently their ratio in (17) does not change sign. Hence, once the initial conditions are fixed, the sign of the function $F$ depends on the relative magnitude of the combinations of parameters $m_{3} /\left(3 M \lambda_{1}\right)$ and $3 m_{2}^{2} /(M \mu)$. The flow of these is depicted in Fig.1 for a small and a large initial value of $\lambda_{1}\left(M^{*}\right)$. The dashed line marks $3 m_{2}^{2} /(M \mu)=m_{3} /\left(3 M \lambda_{1}\right)$ where $F=0$. The region to the left of this line corresponds to the breaking of SU(5) to $G_{S M}$ while the region to the right corresponds to unbroken $\mathrm{SU}(5)$. For all the chosen initial values we 
have checked numerically that the contribution of $\delta_{3 / 2}^{2}$ is indeed negligible over the whole range of the running. The figure clearly displays the attracting fixed point; however, the attraction is typically rather weak between the Planck scale (first mark on the flow), and the GUT scale (third mark on the flow). Interestingly, flows starting on the left (right) of the dashed line stay on the left (right); therefore the flows never cross the boundary between the region of parameter space corresponding to unbroken $S U(5)$ and the one corresponding to $S U(5)$ breaking to $G_{S M}$. This general property implies that the running does not affect the amount of tuning needed for the phenomenologically desirable scenario of $\mathrm{SU}(5)$ breaking to $G_{S M}$, in the sense that the region of parameter space supporting this scenario is mapped into itself by the $\mathrm{RG}$ flow $^{2}$. We conclude that, while it does not require any fine tuning, the scenario with $S U(5)$ breaking to the Standard Model is not a compelling prediction of the infra-red RG structure of this SUSY $S U(5)$ GUT.

\section{A non-SUSY SU(5) model}

Non-SUSY SU(5) with first step of SSB involving a 24 adjoint Higgs was investigated in Ref. [12]. Limiting again our analysis to the Higgs responsible for the first step of SSB, we easily find that the Higgs potential can be written as 13

$$
V(\phi)=-\frac{1}{2} m \operatorname{Tr} \Phi^{2}-\frac{\sqrt{5}}{3 !} f \operatorname{Tr} \Phi^{3}+\frac{5}{4 !} g \operatorname{Tr} \Phi^{4}+\frac{1}{4 !} h\left(\operatorname{Tr} \Phi^{2}\right)^{2}
$$

where $\Phi$ is a traceless $5 \times 5$ matrix.

In the following, we will not consider the case $f=g=0$, since in this case the symmetrybreaking direction is arbitrary (if $f=g=0$ the potential depends only on the norm of the Higgs field). We emphasize that the classification in terms of the cases $f=0, f \neq 0, g=$ $0, g>0$, and $g<0$ is scale invariant [13].

Let us begin assuming $f \neq 0$ and $g=0$. In this case there is only one possibility for the spontaneous symmetry breaking trajectory [13]: $S U(5) \rightarrow S U(4) \otimes U(1)$. At the transition point the function $Q=3 \mathrm{mh} / \mathrm{f}^{2}$ vanishes. Since the derivative of $Q$ does not vanish at this point, the changing of the scale can allow a change of the symmetry from $S U(5)$ to $S U(4) \otimes U(1)$ and vice versa. $Q$ admits a stationary point at $Q=189 / 880$, above which the symmetry is $S U(4) \otimes U(1)$.

For $f=0$ and $g \neq 0$ the breaking direction is independent of the scale and the residual symmetries are $S U(4) \otimes U(1)$ or $G_{S M}$ if $g<0$ or $g>0$ respectively.

The case of interest for our RG naturalness analysis is of course the general case $f, g \neq 0$. The direction of symmetry breaking is found to depend only on the following ratios:

$$
L=\frac{3 m g}{f^{2}}, G=\frac{h+g}{g} .
$$

The corresponding RG equations are:

$$
\frac{d L}{d t}=g\left(\frac{52}{75} L G-\frac{6386}{975} L-\frac{63}{100}\right)
$$

\footnotetext{
${ }^{2}$ This would not be the case if there were significant contributions from $\delta_{3 / 2}^{2}$, since in that case the flow to the unbroken-SU(5) region is favored.
} 
(a)

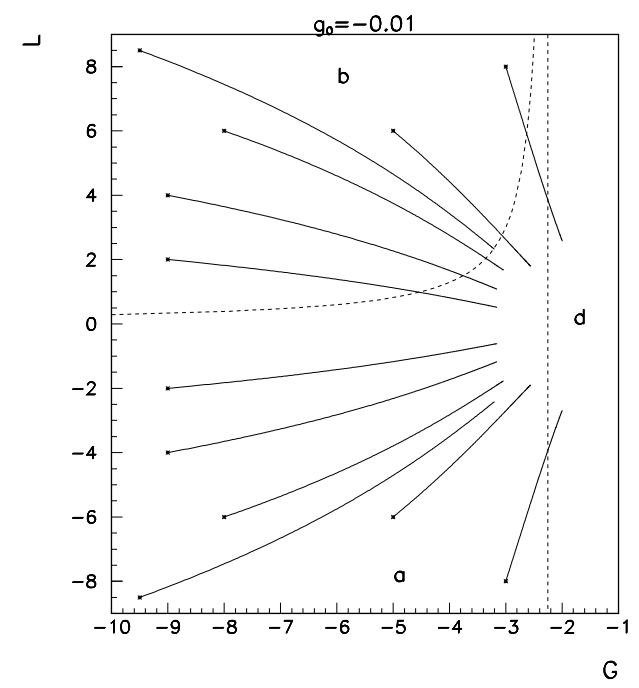

(b)

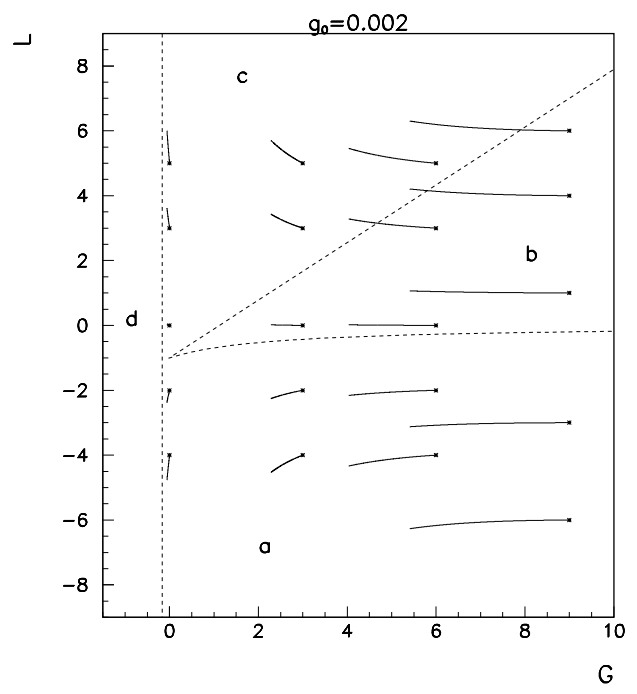

Figure 2: $R G$ flow between $M_{P}$ and $M_{X}$ of the symmetry breaking parameters in non-SUSY $S U(5)$. Regions $\mathbf{a}, \mathbf{b}$, and $\mathbf{c}$ correspond to the symmetry groups $S U(5), S U(4) \otimes U(1)$, and $G_{S M}$ respectively. In region $\mathbf{d}$ the potential is unbounded from below. Also notice that the initial condition (at $M_{P}$ ) is marked on the flow.

$$
\begin{aligned}
\frac{d G}{d t} & =g\left(\frac{56}{25} G^{2}+\frac{652}{195} G+\frac{28558}{12675}\right) \\
\frac{d g}{d t} & =g^{2}\left(\frac{8}{25} G+\frac{8}{15}\right)
\end{aligned}
$$

The RG flows, for $L$ and $G$, are depicted for the two cases $g_{0} \equiv g\left(M^{*}\right)=0.002$ and $g_{0}=-0.01$, with $M^{*}=M_{P}$. These two cases ${ }^{\beta}$ are representative of the two typical situations $g_{0}<0$ and $g_{0}>0$. In both figures the dominant qualitative behavior is the one associated to the fixed point $L=0, G \rightarrow \infty$. This fixed point can be seen directly from the Eqs. (20)-(22) by observing that for $g=0$ the right-hand side of this system of coupled equations vanishes. The limit $L=0, G \rightarrow \infty$ follows from $g=0$ as a result of Eq. (19). As seen from Fig.2 the fixed point is infra-red stable when $g_{0}<0$, whereas for $g_{0}>0$ it gives a saddle point.

Let us now discuss the implications for symmetry breaking of this RG analysis, starting with the case $g_{0}=-0.01$. Here the $G$ values are restricted to the $(-\infty,-9 / 4)$ interval, otherwise the potential becomes unbounded from below. The vertical dashed line in Fig.2 2 a corresponds to $G=-9 / 4$ and separates this region (d) from the other ones. If $L>-1 /(1+$ $4 G / 9)$ the symmetry group is given by $S U(4) \otimes U(1)$ (region b), while in the opposite case there is no symmetry breaking at all (region a). From the figure we see that if $L<-1 /(1+$ $4 G / 9)$ the curves remain in the $S U(5)$-symmetric region, but in some cases they end up in d-region. On the other hand, we observe that when the flows start with $L>-1 /(1+4 G / 9)$ (i.e. they start from the b-region) they quickly come out of the region of $S U(4) \otimes U(1)$ symmetry and end up in the $S U(5)$-symmetric region. We conclude that for $g_{0}<0$ the breaking of $S U(5)$ appears to be quite unnatural in light of the results of the RG analysis, even though the non-dynamical part of the analysis presented us with a symmetry-breaking (b) region of size roughly comparable to the one of the symmetric-preserving (a) region.

\footnotetext{
${ }^{3}$ The very small values of $\left|g_{0}\right|$ shown in Fig. 2 have been chosen because they allow to illustrate more clearly the qualitative structure of the RG running, which only depends on the sign of $g_{0}$. In any case one should in principle only consider $g_{0} \ll 1$ so that our one-loop equations are reliable.
} 
In the case $g_{0}>0$ the $(L, G)$ plane is divided into three regions, a, b, and $\mathbf{c}$, which correspond to the symmetries $S U(5), S U(4) \otimes U(1)$, and $G_{S M}$ respectively. Note that for $G<-1 / 6$ the potential is unbounded from below and, once again, in Fig.2p the vertical dashed line at $G=-1 / 6$ separates the d-region from the other ones. Importantly, the curves that originate in the $\mathbf{c}$ region remain in that part of the plane, whereas the flows with starting point in the b-region rather quickly (depending on how close the starting point is to the line $L=-1+8 G / 9)$ end up in the c-region. This implies that for $g_{0}>0$ the breaking of $S U(5)$ to $S U(4) \otimes U(1)$ is quite unnatural, whereas the breaking of $S U(5)$ to $G_{S M}$ does not require any fine-tuning. Actually, as a result of the fact that some of the flows starting in the b-region end up in the c-region, the RG flow maps a larger portion of parameter space at the scale $M^{*}$ into the c-region of parameter space, which corresponds to the breaking of $S U(5)$ to $G_{S M}$. However, this portion of Higgs parameter space that corresponds to the "phenomenologically reasonable" symmetry breaking to $G_{S M}$ is not a general attractor for the RG flow; in fact, flows starting in the a-region remain in that region, so that the case of unbroken $S U(5)$ is also quite consistent with our RG analysis. Still, as the present article is searching for qualitative structures that might in general characterize this type of RG analyses it is interesting to notice that even in nonSUSY $S U(5)$ there are scenarios in which the regions of parameter space supporting symmetry breaking are stable with respect to the ones supporting unbroken $S U(5)$; in fact, there is no flow across the $L=-1 /(1+4 G / 9)$ curve.

For completeness, in closing this section let us comment on the fact that in Fig.2 there is an approximate symmetry with respect to the $L \rightarrow-L$ exchange. This reflects the fact that the Eqs. (20)-(22) are invariant with respect to the $L \rightarrow-L$ exchange when $L$ and $G$ are large enough that one can ignore the factor $-63 / 100$.

\section{A non-SUSY SO(10) model}

Non-SUSY SO(10) with first step of SSB involving a $\underline{54}$ Higgs was investigated in Refs. 5 , 14, 15]. Besides the $\underline{54}$, the Higgs sector of the model also includes $\underline{10}+\underline{126}+\underline{126}{ }^{*}$ Higgs plus $3 \times 16$ representations containing the SM fermions and 3 right-handed neutrinos. Again, due to the hierarchy among the scales involved and the fact that we are here concerned only with the first step of SSB, we only consider the Higgs potential involving the Higgs responsible for the first step of SSB. The most general Higgs potential constructed from a $\phi \sim \underline{54}$ Higgs may be written as [5]

$$
V(\phi)=\lambda \operatorname{Tr}\left[\left(\phi^{2}-\frac{1}{10} \operatorname{Tr}\left(\phi^{2}\right)+a \phi\right)^{2}\right]-\lambda b^{2} \operatorname{Tr}\left(\phi^{2}\right)+\frac{\mu}{60}\left[\operatorname{Tr}(\phi)^{2}\right]^{2} .
$$

and the $S O(10)$-invariance may be spontaneously broken to $\mathrm{SO}(\mathrm{m}) \otimes \mathrm{SO}(10-\mathrm{m})$ with $m=$ $1,2,3,4,5$ depending upon the combinations of parameters $b^{2} / a^{2}$ and $\mu / \lambda$. Of course, the "phenomenologically reasonable" region of Higgs parameter space is the one that supports symmetry breaking of $S O(10)$ to $S O(6) \otimes S O(4)$, which contains the Standard Model gauge group. In the following we shall denote with $\alpha, \beta, \gamma, \delta$, and $\sigma$ respectively the regions of Higgs parameter space that correspond to the breaking of $S O(10)$ to $S O(5) \otimes S O(5)$, $S O(6) \otimes S O(4), S O(7) \otimes S O(3), S O(8) \otimes S O(2)$ and $S O(9)$. As it will not be visible in our figures we will simply refer to the region of parameter space corresponding to unbroken $S O(10)$ as the $S O(10)$-region.

The radiative corrections to the Higgs potential were examined in Ref. [5] and regions of parameter space yielding "stable minima" (in the specific sense of [5]) were searched for. The objective of our approach is somewhat different in that we investigate the running between 
(a)

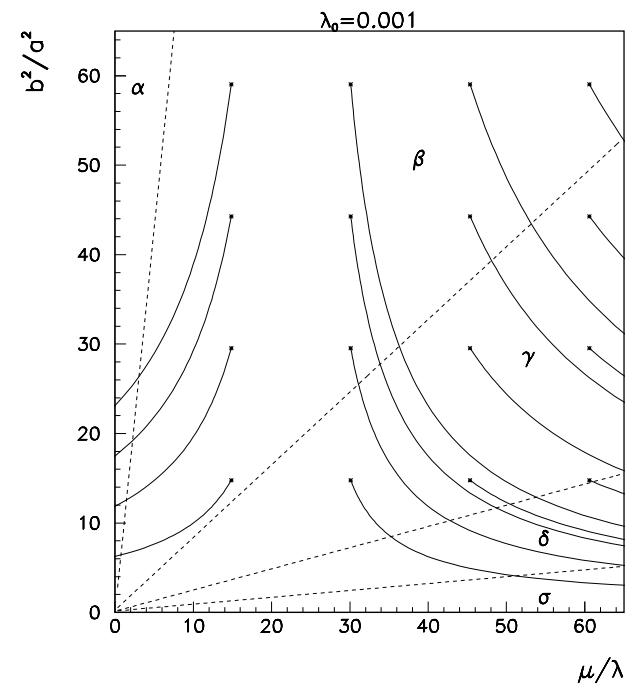

(b)

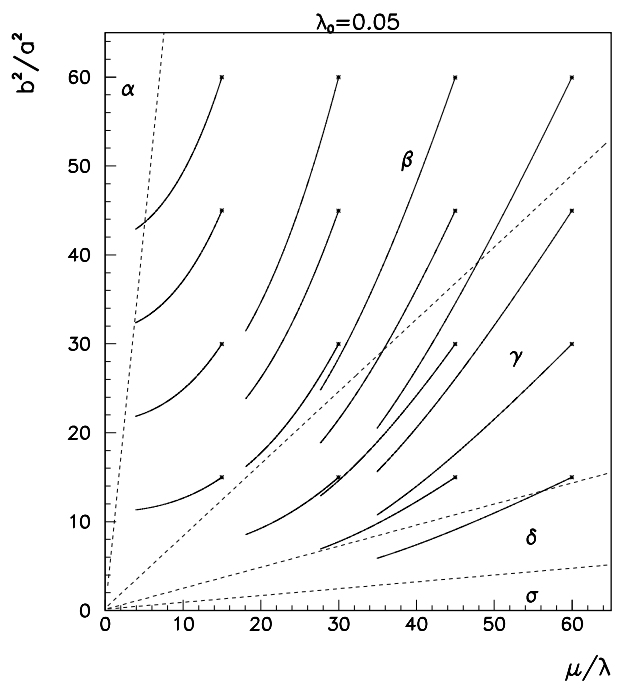

Figure 3: $\quad R G$ flow between $M_{P}$ and $M_{X}$ of the symmetry-breaking parameters in nonSUSY SO(10). The regions $\alpha, \beta, \gamma, \delta$, and $\sigma$ correspond to the breaking of $S O(10)$ to $S O(5) \otimes S O(5), S O(6) \otimes S O(4), S O(7) \otimes S O(3), S O(8) \otimes S O(2)$ and $S O(9)$ respectively. In the parametrization here adopted the region of parameter space corresponding to unbroken $S O(10)$ is located below the $\sigma$-region but it is too small to be seen in figure. Also notice that the initial condition (at $M_{P}$ ) is marked on the flow.

some high scale $M^{*}$ and the GUT scale $M_{X}$ in order to find the most natural intermediate symmetry group, i.e. the most natural group of residual symmetry after the first (GUT-scale) step of SSB. The relevant $\mathrm{RG}$ equations are [5]

$$
\begin{aligned}
16 \pi^{2} \frac{d \lambda}{d t} & =48 \lambda\left(\frac{\mu}{60}-\frac{\lambda}{10}\right)+\frac{508}{35} \lambda^{2}+\frac{15}{2} g^{4}-60 g^{2} \lambda \\
16 \pi^{2} \frac{d}{d t}\left(\frac{b^{2}}{a^{2}}\right) & =\lambda\left[\left(\frac{b^{2}}{a^{2}}-1\right)\left(-\frac{342}{5}+\frac{56}{15} \frac{\mu}{\lambda}+\frac{15}{2} \frac{g^{4}}{\lambda^{2}}\right)-\frac{504}{5}\right] \\
16 \pi^{2} \frac{d}{d t}\left(\frac{\mu}{\lambda}\right) & =\lambda\left[\frac{1264}{5}-\frac{6}{5} \frac{\mu}{\lambda}+\frac{10}{3}\left(\frac{\mu}{\lambda}\right)^{2}+\frac{15}{2} \frac{g^{4}}{\lambda^{2}}\left(24-\frac{\mu}{\lambda}\right)\right] \\
16 \pi^{2} \frac{d g^{2}}{d t} & =-\frac{70}{3} g^{4} .
\end{aligned}
$$

We have been unable to find combinations of parameters allowing the identification of a fixed point, and therefore we proceed directly to a numerical approach.

Here we work with $g^{2}\left(M_{X}\right)=(4 \pi) / 42$. Fig. 3 shows the renormalization group flow for the parameters relevant for symmetry breaking, $\mu / \lambda$ and $b^{2} / a^{2}$, for various choices of initial conditions at $M^{*}=M_{P}$. The figures clearly show that there are regions of attraction for the flow of the couplings. In the case of large initial values of $\lambda$ there is a strong attraction to the $\mathrm{SO}(5) \times \mathrm{SO}(5)$ region of parameter space. With decreasing $\lambda\left(M^{*}\right)$ this attraction becomes weaker until, at about $\lambda\left(M^{*}\right) \approx 0.03$, a new feature emerges: a dividing line which is not crossed. Everything to the right of this line is attracted to the $S O(9)$ - and $S O(10)$-invariant regions, while everything to the left of this line is still attracted toward the $S O(5) \otimes S O(5)$ invariant region. The region of parameter space leading to "phenomenologically reasonable" symmetry breaking, i.e. the one corresponding to intermediate symmetry $S O(6) \otimes S O(4)$, 
does not appear to require large fine tuning, although the RG flow maps a smaller portion of parameter space at the scale $M^{*}$ into the "phenomenologically reasonable" region of parameter space.

\section{Cosmology and Supercosmology}

In the previous sections we have concentrated on a zero-temperature analysis. However, clearly an important constraint on GUTs is the consistency with a working cosmological scenario, and checking this consistency requires in general a finite-temperature analysis. While we postpone this type of analysis to future work, in this section we make a few observations concerning the cosmological relevance of studies of the type reported in the previous sections.

We observe that there is a substantial difference between the SUSY and the non-SUSY cases. In non-SUSY GUTs the potential-energy difference between the absolute minimum of the Higgs potential and other minima is of order the GUT scale. This implies that at temperatures of order $M_{X}$, i.e. once the temperature is low enough that the features of the zero-temperature effective potential become relevant (at high temperatures thermal effects dress the potential in such a way that the absolute minimum is necessarily symmetric [16]), the universe rapidly reaches the vacuum corresponding to the absolute minimum of the zero-temperature effective potential. Therefore for non-SUSY GUTs an analysis of the type presented in the previous sections is directly relevant for the understanding of certain cosmological issues, such as the the selection of the presently observed vacuum.

An important factor affecting the corresponding analysis of SUSY GUTs is the near degeneracy (up to SUSY-breaking terms) of several minima, which we mentioned in Sec.2. The energy difference between the absolute minimum and the other minima is of order SUSY-breaking terms, and therefore much smaller than the GUT scale. As a result, at least within a perturbative analysis, one finds that even when the temperature becomes low enough for the features of the zero-temperature effective potential to become relevant, the universe does not rapidly reach the vacuum corresponding to the absolute minimumf of the zero-temperature effective potential [18. Actually, quick estimates within ordinary perturbative approaches are sufficient to show that the time needed for the transition to the true vacuum should be expected to be longer than the lifetime of the universe [18].

One way to obtain working supercosmology [19, 20] scenarios is to advocate strongcoupling [20] thermal effects, which are indeed at work in the SUSY case [21]. The investigation of these issues requires a careful (and very delicate) thermal analysis which goes beyond the scope of this article. However, it should be noticed that the type of analysis given in Sec.2 is not very relevant to this type of supercosmological issues.

A more conventional (but ad hoc) way to obtain working supercosmological scenarios is based [22] on fine tuning of the parameters of the Higgs potential. One scales down the entire superpotential, so that the height of the potential barrier between competing vacua becomes of the same order of their energy difference, while keeping fixed the mass of the gauge bosons mediating proton decay. For example in the minimal SUSY SU(5) GUT

\footnotetext{
${ }^{4} \mathrm{~A}$ recent analysis by Abel and Savoy 17 of charge and color breaking (CCB) minima in the MSSM showed that a global CCB minimum is neither necessary nor sufficient for symmetry breaking. Abel and Savoy then concentrated on a sufficient condition for no symmetry breaking, namely that the radiatively corrected potential not contain a local CCB minimum. Analyses such as the one we perform in the present article does not provide insight in issues relevant for cosmological studies of the type reported in Ref. [17, since we focus on the identification of the global minimum in the zero temperature potential.
} 
discussed in Sec.2 one would divide [22] both $\lambda$ and $\mu$ by a common (so that the ratio $\mu / \lambda$ giving mass to the gauge bosons mediating proton decay remains unchanged) large factor, of order $10^{12}$. Analyses of the type advocated in the present paper could be relevant for this supercosmology scenario; one can in fact check the level of fine tuning at the Planck scale needed to have a $10^{-12}$ fine tuning at the GUT scale. We find that the fine-tuned values of $\lambda$ and $\mu$ are so far from the region of attraction of the fixed point that the RG running between $M_{P}$ and $M_{X}$ is not substantial: a fine tuning of $10^{-13}$ is required at $M_{P}$ in order to obtain a $10^{-12}$ fine tuning at the GUT scale.

\section{Closing Remarks}

The three GUTs that were considered in the previous sections have allowed us to illustrate various possibilities for the outcome of RG naturalness analyses, although a common property of these illustrative examples is that one does not find a compelling SSB scenario compatible with the low-energy phenomenology of the Standard Model. This could be interpreted positively, since these three GUTs are already known to give rather unsatisfactory predictions for low-energy observables, f even when their SSB pattern is tuned (by tuning the parameters of the Higgs potential) to be group-theoretically consistent with the Standard Model. One is tempted to hope that applying the same naturalness criterion to the GUTs that are already known to have good low-energy phenomenology it would be possible to find one for which even the SSB pattern appeared compelling (i.e. such that the values of the parameters of the Higgs potential that correspond to this SSB pattern could be obtained via RG running from rather generic input parameters at the scale $M^{*}$ ). Models with enough structure to be consistent with low-energy phenomenology will require rather complicated RG naturalness analyses from the technical point of view, but the conceptual steps are of course just the ones discussed here.

Among the properties illustrated by our three simple illustrative examples, particularly significant is the fact that in all the three cases we find that the RG naturalness criterion appears to favor spontaneous-symmetry-breaking $G \rightarrow H$ scenarios such that the residualsymmetry group $H$ is the "smallest" (the one with the smallest number of generators) of the maximal little groups of the group $G$ which is being broken. In fact, both in the SUSY and in the nonSUSY cases with $S U(5)$ grand unification group the breaking of $S U(5)$ to $S U(4) \otimes U(1)$ was quite unnatural in all scenarios considered, whereas under appropriate conditions the breaking of $S U(5)$ to $G_{S M}$ (which is the smallest of the maximal little groups of $S U(5))$ appeared to be as natural as the possibility that $S U(5)$ would remain unbroken. Similarly, in our analysis of the first step of SSB of a nonSUSY $S O(10)$ model we found that under certain conditions the breaking of $S O(10)$ to $S O(5) \otimes S O(5)$ (which is the smallest of the maximal little groups of $S O(10)$ ) appeared to be as natural as the possibility that $S O(10)$ would remain unbroken, while all other possibilities appeared to be quite unnatural from the RG viewpoint (which is quite unfortunate since $S O(5) \otimes S O(5)$ does not contain the Standard Model gauge group). It appears that the RG running favors either the maximal preservation of symmetries (unbroken $G$ ) or the maximal breaking of symmetries (which in a specific sense corresponds to the breaking of $G$ to its smallest maximal little group). It would be quite interesting to analyze from the point of view of RG naturalness some of the known counterexamples to Michel's conjecture, i.e. GUTs whose Higgs potential have enough structure to allow breaking to a non-maximal little group (see, e.g., the example discussed in Ref. [15]).

\footnotetext{
${ }^{5}$ For example, the minimal SUSY $S U(5)$ GUT does not address the question of doublet-triplet splitting [23], while the nonSUSY $S U(5)$ and $S O(10)$ GUTs here considered are not consistent with the available data on proton stability (see, e.g., Ref. [15]).
} 
Our findings also suggest (as it was to be expected) that there are many technical differences between the RG naturalness analysis of a SUSY GUT and the corresponding analysis of a nonSUSY GUT. For example, the simplifications associated to SUSY allow one to find analytically a fixed point in the case of SUSY $S U(5)$.

While we focused on the first step of SSB in our discussion and examples, the criterion of RG naturalness can obviously be applied to any of the steps of a SSB pattern. Actually, in some cases the criterion (at least as applied in the present article) might be least meaningful when considering the first step of SSB. In fact, it appears plausible that only two or three orders of magnitude would separate the scale $M^{*}$ from $M_{X}$, in which case omitting non-renormalizable terms, as we have done above, might be unjustified. Several orders of magnitude instead should separate the GUT scale $M_{X}$ from the scale $M_{I I}$ of the second step of SSB, which could be the electroweak scale or (in models with an intermediate symmetry group [15]) a scale of order $10^{9} \sim 10^{12} \mathrm{GeV}$. Therefore, most practical applications of the ideas here presented might end up being found in studies of the running between $M_{X}$ and $M_{I I}$, for which non-renormalizable terms can be safely ignored, rather than the running between $M^{*}$ and $M_{X}$ which we have here considered for illustrative purposes.

Perhaps the most robust observation one can make based on the results here reported is that the conventional tests of the naturalness of a GUT are quite inadequate. According to these conventional naturalness tests one should disregard GUTs in which a fine tuning of the Higgs parameters is needed in order to realize a phenomenologically acceptable SSB pattern. However, viewing the GUTs as effective low-energy descriptions of a more fundamental theory one would like to check whether the phenomenological SSB pattern corresponds to fine tuning of the Higgs parameters at the scale $M^{*}$. Some of our results indicate that it is not uncommon that a scenario requiring no fine tuning of the Higgs parameters at $M^{*}$ might correspond via the RG running (for example in presence of an appropriate infra-red fixed point) to a narrow region (apparent fine tuning) of the Higgs parameter space at $M_{X}$, where the first step of SSB is decided. (Of course, corresponding statements should apply to the other steps of the SSB pattern.) Our analysis also provided examples of the opposite, i.e. a SSB pattern that in a "conventional naturalness test" would appear to correspond to a significant portion of the Higgs parameter space actually requires some level of fine tuning at $M^{*}$, since the corresponding portion of Higgs parameter space is "disfavored" by the RG running.

\section{Acknowledgments}

We have greatly benefited from conversations with G. Ross, which we happily acknowledge. One of us (G.A.-C.) also thanks K. Tamvakis for useful discussions. The work of G.A.-C. was supported by a grant of the Swiss National Science Foundation, by a European Union TMR-network grant (no. FMRXCT960045), and by an OFES grant (no. 95.0856) of the Swiss Federal Office for Education and Science.

\section{References}

[1] G. Amelino-Camelia, hep-ph/9610298 (to be published in the proceedings of the 9th International Seminar on High-energy Physics: Quarks 96, Yaroslavl, Russia, 5-11 May 1996).

[2] B.C. Allanach, G. Amelino-Camelia, and O. Philipsen, Phys. Lett. B393 (1997) 349. 
[3] B. Pendleton and G.G. Ross, Phys. Lett. B98 (1981) 291; M. Lanzagorta and G.G. Ross, Phys. Lett. B349 (1995) 319; Phys. Lett. B364 (1995) 163; G.G. Ross, Phys. Lett. B364 (1995) 216; S.A. Abel and B.C. Allanach, Phys. Lett. B415 (1997) 371; hep-ph/9803476; B.C. Allanach and S.F. King, Phys. Lett. B407 (1997) 124; Nucl. Phys. B407 (1997) 275; G.G. Ross and S.F. King, hep-ph/9803463.

[4] J. Basecq, S. Meljanac, and L. O’Raifeartaigh, Phys. Rev. D39 (1989) 3110.

[5] M. Abud, F. Buccella , A. Della Selva, A. Sciarrino, R. Fiore, and G. Immirzi, Nucl. Phys. B263 (1986) 336.

[6] See, for example, C. Lucchesi, O. Piguet, and K. Sibold, Phys. Lett. B201 (1988) 241; D.I. Kazakov, M.Yu. Kalmykov, I.N. Kondrashuk, and A.V. Gladyshev, Nucl. Phys. B471 (1996) 389.

[7] L. Ibanez and G.G. Ross, Phys. Lett. B110 (1982) 215.

[8] F. Buccella, J.P. Derendinger, S. Ferrara, C.A. Savoy, Phys. Lett. B115 (1982) 375; N. Dragon, Zeit. Phys. C15 (1982) 169; G.A. Diamandis and B.C. Georgalas, Phys. Lett. B160 ( 1985) 263; M. Drees, S. Meljanac, M. Milekovic, and S. Pallua, Phys. Lett. B178 (1986) 226.

[9] H. E Haber, hep-ph/9306207 (TASI-92 Lectures).

[10] S. P. Martin and M. T. Vaughn, Phys. Rev. D50 (1994) 2282.

[11] I. Jack and D.R.T. Jones, Phys. Lett. B349 (1995) 294.

[12] L. Li, Phys. Rev. D9 (1974) 1723.

[13] T. Murphy and L. O'Raifeartaigh, Nucl. Phys. B229 (1983) 509; T. Murphy, L. O’Raifeartaigh, and M. Yamada, Nucl. Phys. B248 (1984) 365.

[14] Q. Shafi and C. Wetterich, Phys. Lett. B85 (1979) 52; F. Buccella, L. Cocco, and C. Wetterich, Nucl. Phys. B243 (1984) 273.

[15] F. Acampora, G. Amelino-Camelia, F. Buccella, O. Pisanti, L. Rosa, and T. Tuzi, Il Nuovo Cimento A108 (1995) 375.

[16] G. Amelino-Camelia, hep-ph/9503451 (in the proceedings of Trends in Astroparticle Physics, Stockholm, Sweden, 22-25 Sep 1994); Phys. Lett. B388 (1996) 776; Nucl. Phys. B476 (1996) 255.

[17] S.A. Abel and C.A. Savoy, hep-ph/9803218.

[18] M. Srednicki, Nucl. Phys. B206 (1982) 132.

[19] M. Srednicki, Nucl. Phys. B202 (1982) 327; D.V. Nanopoulos and K. Tamvakis, Phys. Lett. B110 (1982) 449; J. Ellis, C.H. Llewellyn Smith, and G.G. Ross, Phys. Lett. B114 (1982) 227. J. Ellis, D.V. Nanopoulos, K.A. Olive, and K. Tamvakis, Phys. Lett. B118 (1982) 335.

[20] D.V. Nanopoulos, K.A. Olive, and K. Tamvakis, Phys. Lett. B115 (1982) 15.

[21] H. Goldberg, Phys. Lett. B139 (1984) 45; J. Kapusta, D.B. Reiss, and S. Rudas, Nucl. Phys. B263 (1986) 207. 
[22] M. Milekovic and S. Pallua, Phys. Rev. D36 (1987) 2874.

[23] R. Barbieri, G. Dvali and M. Moretti, Phys. Lett. B 312 (1993) 137. 